\title{
MODELING OF SUBSTRATE NOISE INJECTED BY DIGITAL LIBRARIES
}

\begin{abstract}
Switching noise is one of the major sources of timing errors and functional hazards in logic circuits. It is caused by the cumulative effect of microscopic spurious currents present in all devices during logic transitions. These currents are injected into the substrate and in supply lines, resulting in significant ripple noise. Individually, such micro-currents, do not usually cause catastrophic failures. However, cumulatively, they can impact power supply and substrate potential across the chip. Thus, the electrical behavior of sensitive digital and analog circuits can be significantly changed, hence limiting circuit performance. The analysis of switching noise at a macroscopic level requires one to accurately compute models for all microscopic spurious currents, known as noise signatures. The challenge is to simultaneously account for a myriad of parameters and their process variations in a compact and accurate model. To address this problem, a new methodology based on Response Surface Methodology and Orthogonal Polynomials approximation is being proposed. Experimental results on a $0.35 \mu \mathrm{m}$ library show that the methodology is capable of accurately approximating noise signatures with a single analytical formula. A library of such formulae has been created and it is being used to accurately characterize switching noise at a macroscopic level.
\end{abstract}

\section{INTRODUCTION}

Almost every aspect of today's integrated electronics aimes to meet tougher performance budgets, while continuously shrinking device features and power constraints. An aggressive reduction of feature sizes often results in lower signals, higher noise, which cause lower noise margins, hence higher potential functionality hazards and/or lower performance.

There exists two main types of noise with major effects to performance: intrinsic and switching noise. Intrinsic noise relates to fundamental physical phenomena underlying electrical conduction. Because of the inherent noise robustness of the digital encoding, the impact on digital circuits is usually limited. Hence, the odds that a transition could be disrupted are relatively low. Switching noise is the macroscopic effect resulting from microscoping currents being injected during a logical transition or glitch. Individually, such spurious currents rarely cause catastrophic faults, however, cumulatively, they can significantly impact timing, power and even functionality.

Switching noise is generally present in supply and ground lines, as well as substrate taps in form of absorbed/injected current spikes which generally follow a relatively stable pattern. Such current patterns can have a detrimental impact on the injecting circuit, as well as on other digital and ana$\log$ blocks in the proximity. The effect on analog blocks can be particularly destructive, due to the sensitivity of such circuits to small changes in bias voltage.

A very coarse estimate of switching noise has been proposed in the literature as a capacitively and/or resistively coupled current or voltage generator whose waveform is derived from the circuit's global clock [1], [2]. One of the limitations of this approach is the lack of accuracy of the estimate. In fact a significant portion of the energy spectrum of switching noise resides outside the harmonics of the clock. This is due to the fact that every device contributes to switching noise in a unique way, thus producing a complex noise waveform that needs be modeled in detail.

One way to properly characterize switching noise is to perform a detailed device-based simulation of the entire circuit. However, in most cases, simulating every spurious current in the circuit can be extremely time consuming if at all possible. A way to cope with this problem is to construct models of the current absorbtion/injection pattern. Such pattern is called noise signature and it is computed for each gate or device, independently. The waveform produced by the model is then convolved with the transition activity of every standard cell or device and the switching noise is computed by adding all the resulting signals into a global injection signature [3]. This signature is a time domain representation of the noise, which can be further processed to produce an equivalent frequency domain spectrum as suggested in [4] and more recently in [5].

The main limitation of these approaches is the lack of a realistic model for the noise signatures, which, to be accurate, require knowledge of the details of device and standard cell design, including loading, input stimuli, power supply, process technology variations, etc. As an illustra-

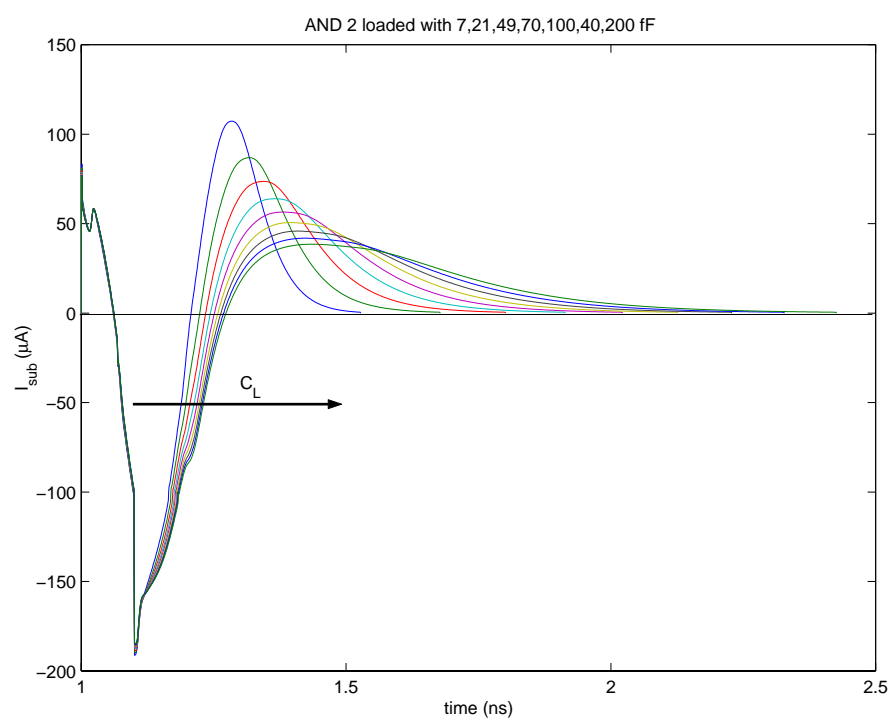

Fig. 1. Signature of a gate loaded with different capacitance values

tion, consider Figure 1. The waveform represents the noise signature of a gate connected to a variety of loads. The variation of the carriers injected into the substrate can be 
significant even when small variations of the input stimuli and load occur, thus potentially impacting the global switching noise.

In order to address this fundamental limitation, in this paper we propose a flexible and compact modeling technique to characterize large digital libraries. The technique is designed to account for very large collections of deterministic and non-deterministic parameters in a large range of combinations, far superior to the standard four-corner analysis without the computational handicap of Monte Carlo methods. Complex standard cells can be characterized with high levels of accuracy using relatively simple analytical models which cover a large set of statistical experiments. The analytical models can then be efficiently used for the computation of switching noise in large systems.

The method consists of the following steps. First, using Response Surface Methodology (RSM), the moments of the noise signature of a certain standard cell are computed. Second, an analytical model of the signature is generated using a Legendre approximation of the collection of samples, so as to minimize the modeling error. Finally, model is verified and the accuracy of the approximation is computed.

Due to the fundamental properties of Legendre polynomials, one can show that convergence can always be obtained provided that certain assumptions hold. Furthermore, the model is extremely compact making it an excellent candidate for the evaluation of switching noise. Extensive experimentation was conducted on a large set of industrial examples implemented in a $0.35 \mu \mathrm{m}$ CMOS technology to verify the effectiveness of the methodology. The examples show the versatility of the method especially in the evaluation of noise signatures under various fabrication regimes.

The paper is organized as follows. Section II describes RSM and its propterties in light of the current application domain. Section III outlines the library characterization methodology as a whole. Section IV presents experiments showing the suitability of the methodology.

\section{Response Surface Methodology and Orthogonal Polynomials}

In this section we will briefly review the mathematical formalism used in the following sections.

\section{A. Response Surface Methodology}

RSM[6] is a well known empirical tool for building polynomial macromodels of physical systems subject to multiple inputs. In order to measure the accuracy of the models, several metrics have been developed. Among them, $R_{a d j}^{2}$ measures the lack of fit of the model, weighted by the number of polynomial terms. A value close to 1 indicates a good fit. In general, accurate models achieve 0.95 or higher $R_{a d j}^{2}$.

In practice, the response surfaces are Taylor expansions of the system output. The partial derivatives are computed by running some experiments (in our case circuit simulations) rather than computing them by numerical analysis.
RSM is essentially composed of two steps: the choice of the model and the execution of the experiments. In modeling integrated circuits, low (often second) order polynomials usually provide a satisfactory fit. An intuitive reason for this is that the goal of process and circuit designers, is to achieve low sensitivity to environmental and process variables. If this were not the case, small changes in some variables would cause large variations in performance metrics, which is generally not desirable.

In order to achieve a high degree of accuracy and limit the number of simulations necessary to fit the model, the Design of Experiments must be carefuly hosen. In our work we will use the Central Composite Design (CCD). CCD[7] is a DOE particularly suited for quadratic models. It yields an unbiased estimation of all the terms of a second order model. CCD requires a $2^{n-k}+2 n+1$ simulations, where $\mathrm{n}$ is the number of variables and $\mathrm{k}$ is a function of $\mathrm{n}$. After the simulation, the model is estimated by using multivariate least square regression [8].

\section{B. Orthogonal Polynomials}

The signature of a digital circuit, is a continuous and limited function of time. Hence, it is square-integrable. Since continuous, square-integrable functions can be expanded in orthogonal polynomial series, they are suitable for approximating the current injected into the substrate.

In this section a quick overview of the properties of the Legendre Polynomials is presented. Only those relevant to the problems addressed in this paper are illustrated. For further details the reader is referred to [9].

Let $f$ be a function defined on a set $G$. If the integral:

$$
m_{n}=\int_{G} x^{n} f(x) d x, \quad n \geq 0, n \in \aleph
$$

exists, it is called the $n$-th moment of the function $f$.

Let us suppose that the function $f(x)=0, \forall x \notin[a, b]$. Let us also suppose without loss of generality that $[a, b]=$ $[-1,1]$. If this were not the case, an affine transform could be applied.

The polynomials:

$$
L_{n}(x)=\sum_{k=0}^{n} d_{n, k} x^{k} \quad n, k \in \aleph, k \leq n
$$

are called the Legendre Polynomial of order $n$ if and only if $[10]$ :

$$
\begin{array}{rlrl}
(n+1) d_{n+1, k} & =(2 n+1) d_{n, k-1} & -n d_{n-1, k} \\
d_{0,0} & =1 & d_{0, k} & =0, k>0 \\
d_{1,1} & =1 & d_{1, k} & =0, k>1
\end{array}
$$

The Legendre polynomials are mutually orthogonal, i.e. $\int_{-1}^{1} L_{n}(x) L_{m}(x) d x=0$ if and only if $m \neq n$.

Under some loose hypotheses, a function $f$, square integrable in $[-1,1]$ can be expandeded as [11]:

$$
f(x)=\sum_{n=0}^{\infty} c_{n} L_{n}(x) .
$$


The coefficients $c_{n}$ are given by:

$$
c_{n}=\frac{1}{h_{n}} \int_{-1}^{1} f(x) L_{n}(x) d x, \quad n \in \aleph,
$$

where:

$$
h_{n}=\int_{-1}^{1} L_{n}^{2}(x) d x=\frac{2}{2 n-1}, \quad n \in \aleph
$$

Theorem 1: The coefficients of the series expansion are given by:

$$
c_{n}=\frac{1}{h_{n}} \sum_{k=0}^{n} d_{n, k} m_{k} .
$$

Proof:

$$
\begin{aligned}
c_{n} & =\frac{1}{h_{n}} \int_{-1}^{1} f(x) L_{n}(x) d x \\
& =\frac{1}{h_{n}} \sum_{k=0}^{n} d_{n, k} \int_{-1}^{1} f(x) x^{k} d x \\
& =\frac{1}{h_{n}} \sum_{k=0}^{n} d_{n, k} m_{k}
\end{aligned}
$$

Using the theory of the orthogonal polynomials, it is thus possible to directly relate the moments of a function to the coefficients of the series expansion providing a simple and elegant way to approximate such function.

\section{Signature Approximation}

In this section, the characterization of the current injected into the substrate (signature) by every element of a digital library is presented.

In previous works, the events output rising edge and output falling edge are characterized regardless of the input sequence that caused them. Moreover, no dependence on envinronmental variables $\left(V_{D D}, T\right)$, operating conditions $\left(C_{L}, t_{t i n}\right)$ and process variables $\left(V_{t h}, T_{o x}, \Delta L\right.$, etc. $)$ is taken into account. No signature is associated to input sequences that cause no output transition. However, during an input switching event a gate injects a current into the substrate. Depending upon the input sequence, the devices in the cell will follow a unique injection pattern, yielding different signatures. Furthermore, an injection might take place even in the case that the output does not switch (figure 2). Therefore, it is necessary to separately characterize each possible input sequence.

The substrate current is characterized as function of the envinronmental variables, operating conditions and process variables. It is also possible to characterize the current as function of design parameters (such as the width of certain devices). The range of variation of the enviromental and process variables and of the operating conditions is supposed to be available from process characterization and/or design specifications. In the nominal case (no dependance on aforementioned variables), the moments could be estimated performing a SPICE simulation. In fact, they could

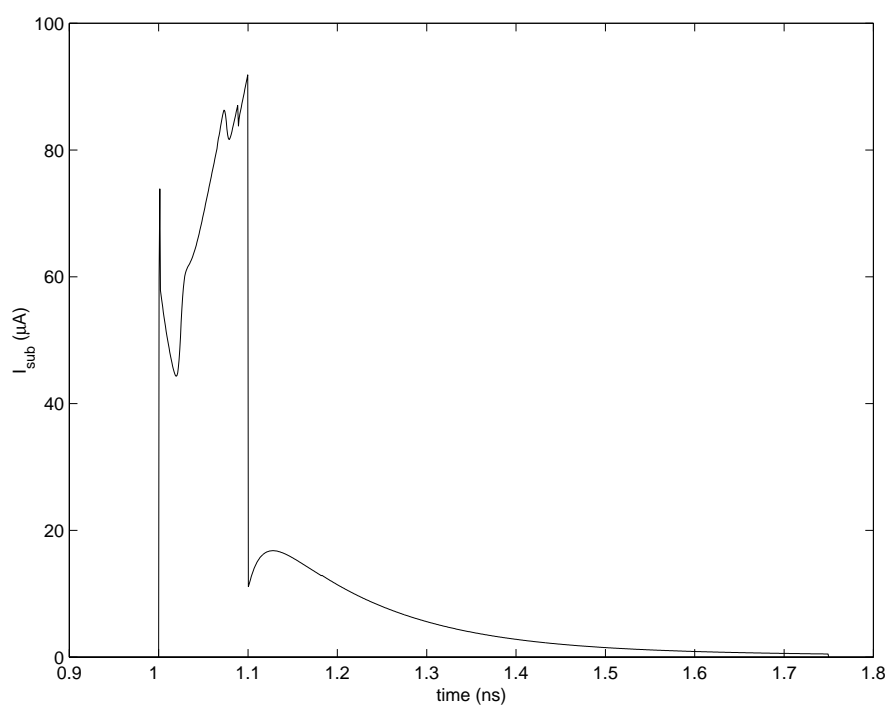

Fig. 2. Signature of an AND2 whose inputs change from 00 to 01

be computed by measuring the integral (1), where $f(x)$ is the substate current $I_{s u b}$ as function of the time $t$. If this were not the case, the moments could be characterized by using the Response Surface Methodology. By runninng a series of SPICE simulations, planned according to a Design of Experiments, the moments of the injected current could be modeled with a (usually) second order polynomial.

The signature of a cell can be represented with a limited function defined in $\left[t_{1}, t_{2}\right]$. According to the theory of orthogonal polynomials, such function can be expanded in series of Legendre Polynomials. In order to determine the coefficients of the series, its moments must be calculated.

The procedure is illustrated in figure 3 . Given the netlist and the characterization range RSM is applied; according to a certain DOE a set of SPICE simulations is run, yielding a polynomial model of each moment is generated. The coefficients of the Legendre series are then computed according to (7). Thus, the resulting series gives an approximation of the injected current as function of operating conditions, environmental and process variables.

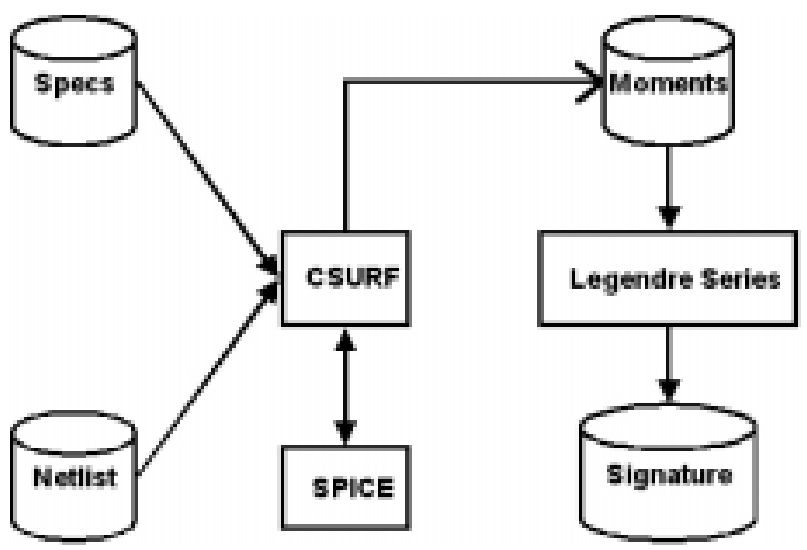

Fig. 3. Signature approximation 


\section{Experimental Results}

The methodology has been applied to a set of gates of a standard cell library designed in a $0.35 \mu m$ technology. The characterization range is reported in table $\mathrm{I}$.

TABLE I

RANGE OF CHARACTERIZATION

\begin{tabular}{|c||c|}
\hline Parameter & Characterization range \\
\hline$V_{D D}$ & $2.5 V \pm 10 \%$ \\
\hline$C_{L}$ & $7-70 \mathrm{fF}$ \\
\hline$t_{t i n}$ & $0.05-2 \mathrm{~ns}$ \\
\hline$V_{t h n}$ & $\pm 3 \sigma$ \\
\hline$V_{t h p}$ & $\pm 3 \sigma$ \\
\hline$\Delta L$ & $\pm 3 \sigma$ \\
\hline$T_{o x}$ & $\pm 3 \sigma$ \\
\hline
\end{tabular}

The first 20 moments of the signatures of the gates have been characterized by using Circuit Surfer[12], a tool capable of statistical design optimization.

The Response Surfarce Methodology has been applied. The characterization required 79 SPICE simulations planned according to a Central Composite Design of Experiments. The average $R_{a d j}^{2}$ is 0.99 , which indicates a very good fit.

The Legendre Series Expansion has been implemented in matlab [13]. After the modeling phase, the accuracy of each model has been tested by randomly generating a sample of 50 different combinations of the characterization variables. For each sample, a SPICE simulation has been run and the root mean square error has been computed and compared to the average of the absolute value of the current (so that negative and positive values don't compensate). The result is reported in table II.

TABLE II

MOdeling ERror

\begin{tabular}{|c||c|}
\hline Cell & Modeling Error \\
\hline INV & $5.05 \%$ \\
\hline AND2 & $8.5 \%$ \\
\hline OAI22 & $6.8 \%$ \\
\hline OR3 & $7.05 \%$ \\
\hline AOI & $4.4 \%$ \\
\hline
\end{tabular}

As an example, the comparison between our methodology and the simulation of an AND2 whose parameters have been randomly chosen is reported in figure 4 . For this case the error is $7.62 \%$.

The modeling error is generally less than 10\%. Experiments suggest that most of the modeling error is due to the nominal case. In fact, in all the cases, the quality of the nominal fit and of the Monte Carlo sample were comparable.

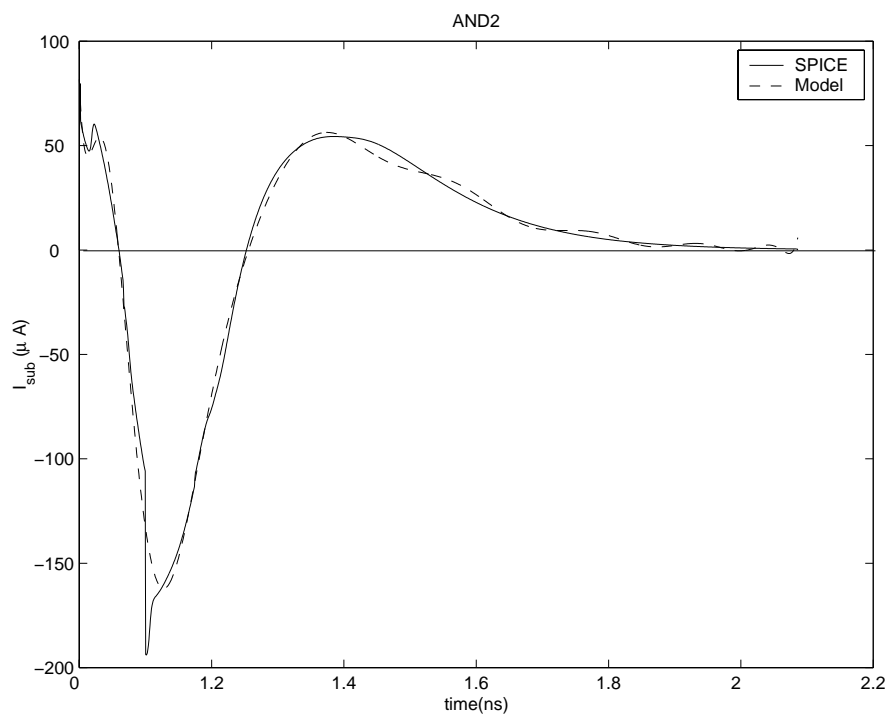

Fig. 4. AND2 whose inputs change from 01 to 11 (error: $7.62 \%$ )

\section{Conclusion}

A methodology for modeling the current injected into the substrate by digital libraries has been proposed. The effect of load, input transition time, supply voltage and process variations is taken into account.

Using RSM the moments of the signatures are modeled as function of the aforementioned variables. The signature is then approximated by a series of orthogonal polynomials. The coefficients of the series are directly derived from the moments. A set of gates from a $0.35 \mu \mathrm{m}$ CMOS standard cell library has been characterized. Each model has been compared to the outcome of 50 SPICE simulations corresponding to 50 points randomly selected in the characterization space, showing the effectiveness of the methodology.

\section{Appendix - Convergence of the Legendre SERIES}

An important issue in function approximation, is to determine under which conditions the series converges and how it convergers (uniform c onvergency is a very desirable property). Mathematically, the problem must be stated carefully, in order to rigorously apply the tools pro vided by the theory of the orthogonal functions. We assume that $I_{\text {sub }} \neq 0 \forall t \in\left[t_{1}, t_{2}\right]$, where $t_{1}$ is the time at which the triggering event takes place, and $t_{2}$ is the time at which the injection terminates. Therefore $I_{\text {sub }}\left(t_{1}\right)=0$ and $I_{\text {sub }}\left(t_{2}\right)=0$. Moreover, $I_{s u b}$ is a continuous and bounded variation function (the former being obvious, the latter coming from the physical nature of a current). It can be shown that under these hypotheses, the series converges uniformly (Hobson's theorem[9], pag. 236).

\section{REFERENCES}

[1] B. R. Stanisic, N. K. Verghese, D. J. Allstot, R. A. Rutenbar, and L. R. Carley, "Addressing substrate coupling in mixed-mode ics: Simulation and power distribution synthesis," IEEE Journal of Solid State Circuits, vol. 29, no. 3, pp. 226-237, March 1994. 
[2] S. Mitra, R. A. Rutenbar, L. R. Carley, and D. J. Allstot, "Substrate-aware mixed-signal macro-cell placement in wright," in Proc. IEEE CICC, May 1994, pp. 529-532.

[3] P. Miliozzi, L. Carloni, E. Charbon, and A. L. SangiovanniVincentelli, "SuBWAVE: a methodology for modeling digital substrate noise injection in mixed-signal ICs," in Proc. IEEE CICC, May 1996, pp. 385-388.

[4] E. Charbon, P. Miliozzi, L.P. Carloni, A. Ferrari, and A.L. Sangiovanni-Vincentelli, "Modeling digital substrate noise injection in mixed-signal ics," IEEE Trans. on CAD, vol. 18, no. 3, Mar. 1999.

[5] A. Demir and J. Roychowdhury, "Modeling and simulation of noise in analog/mixed-signal communication systems," in Proc. IEEE CICC, 1999, pp. 385-93.

[6] G. E. Box and N. R. Draper, Empirical Model-Building and Response Surfaces, J. Wiley \& Sons, New York, 1986.

[7] W. J. Diamond, Practical Design of Experiments, Van Nostrand Reinhold, New York, 1981.

[8] K. Levemberg, "A method for the solution of certain problems nonlinear in least square," Quarth. Appl. Math., vol. 2, pp. 164-168, 1944

[9] G. Sansone, Orthogonal Functions, Interscience, 1959.

[10] I. A. Stegun M. Abramowitz, Handbook of Mathematical Functions, Dover Publications Inc. New York, 1970.

[11] S. Zanella, A. Neviani, B. Franzini, and C. Guardiani, "Statistical timing macromodeling of digital ip libraries," in International Workshop on Statistical Metrology, June 2000.

[12] PDF Solutions Inc., Circuit Surfer User's Manual, February 2000

[13] The Mathworks Inc., Matlab User's Manual, 1999. 